ARTIGO

DOI: http://dx.doi.org/10.20396/sss.v16i1.8651472

(cc) $\mathrm{BY}$

\title{
A INSERÇÃO dE GRUPO dE SALA dE ESPERA COMO PROMOÇÃo EM SAÚDE EM UMA UNIDADE PRÉ-HOSPITALAR
}

\author{
THE INSERTION OF A WAITING ROOM GROUP AS A PROMOTION IN HEALTH IN A \\ PRE-HOSPITAL UNIT
}

\author{
Samantha Cassia da Silva ${ }^{1}$ \\ José Augusto Costa ${ }^{2}$ \\ Isaura Isoldi Castanho de Mello e Oliveira ${ }^{3}$
}

\section{RESUMO}

A partir de um olhar qualificado e sensível sobre as formas de acesso e o entendimento da promoção de saúde, foi possível iniciar uma proposta de intervenção com grupos em sala de espera readequada, abordando os fluxos (Classificação de Risco) e realizando o mapeamento dos serviços de referência e contra referência. $\mathrm{O}$ estudo apresenta uma análise sobre o perfil sócio demográfico dos usuários que frequentam uma Unidade de Urgência e Emergência e o conhecimento que eles apresentam acerca da Classificação de Risco e de sua participação nos serviços de saúde. O intuito desta pesquisa foi encontrar formas de promover saúde com discussões horizontais realizadas em grupos, com a

\footnotetext{
${ }^{1}$ Graduada em Serviço Social pela Universidade Federal de São Paulo, Aprimoranda em Hospital Geral pelo Hospital das Clínicas de São Paulo, com foco em Pronto Socorro. Residente Multiprofissional em Urgência e Emergência (2015-2017) pela PUC São Paulo, Campus Sorocaba. Atualmente trabalha como assistente social no Hospital Modelo de Sorocaba do Grupo Notredame Intermédica, sendo a responsável pelo setor. Telefone: (15) 991382532. E-mail: samanthac.ss @ hotmail.com.

${ }^{2}$ Possui graduação em Medicina, pela Pontifícia Universidade Católica de São Paulo, Mestrado e Doutorado em Medicina pela PUC São Paulo. Atualmente é professor Titular do Departamento de Cirurgia da PUC São Paulo e coordenador do Comitê de Ética em Pesquisa (CEP) da Faculdade de Ciências Médicas e da Saúde da PÚC São Paulo. E-mail: drcosta@globo.com.

${ }^{3}$ Graduada em Serviço Social e Mestrado em Serviço Social pela Pontifícia Universidade Católica de São Paulo. Foi coordenadora do Curso de Serviço Social da Faculdade de Ciências Sociais da PUC SP no período de 2009-2011. É professor assistente mestre do Curso de Serviço Social da Faculdade de Ciências Sociais da PUC SP e pesquisadora da Coordenadoria de Estudos e Desenvolvimento de Projetos Especiais da PUC SP. É uma das coordenadoras do Núcleo de Justiça e Violência e membro do Grupo de Pesquisa de Políticas Públicas para a Violência, cadastrado no CNPq/PUC-UNIFESP-USP. É professora convidada e pesquisadora do Núcleo Trabalho e Profissão do PEPG em Serviço Social da PUC-SP. E-mail: isoldioliveira@ hotmail.com. Serv. Soc. \& Saúde, Campinas, SP v.16, n. 1 (23), p. 25-48, jan./jun. 2017 E-ISSN 2446-5992
} 
construção de conhecimentos de forma coletiva e elaboração de propostas para melhorias durante a passagem na unidade. Foi realizada uma pesquisa quantitativa com a aplicação de questionário sócio demográfico e um questionário estruturado e analisado com a escala Likert, construído pela autora, abarcando questões relevantes à rotina do serviço. Após a aplicação do questionário foram formados grupos, durante um mês, nos três períodos do dia, como forma de abarcar diversos perfis de usuários e auxiliar na construção de conhecimentos e orientações referentes à saúde, pautadas também no Projeto de Reforma Sanitária e no cotidiano do Sistema Único de Saúde. O estudo foi realizado a partir da metodologia da Pesquisa Exploratória que teve como objetivo proporcionar maior privacidade com a questão levantada, possibilitando a construção de hipóteses.

PALAVRAS-CHAVE: Promoção da Saúde. Centros de Saúde. Urgência e Emergência. Serviço Social.

\section{ABSTRACT}

Based on a qualified and sensitive look about the ways of accessing and the understanding of health promotion, it was possible to initiate a proposal of interventions with groups in waiting rooms, addressing the existing flows (Risk Classification) and mapping the reference services and against reference. The study presents an analysis of the socio-demographic profile of users attending Emergency Room Units and the knowledge they present about the Risk Classification and on their participation in health services. The objective of this research was to find ways to promote health through horizontal discussions in groups, with the construction of knowledge in a collective way and elaboration of proposals for improvements during the passage in the unit. A quantitative research was carried out with the application of a socio-demographic questionnaire and with one structured and analyzed questionnaire with the Likert scale, built by the author, covering issues relevant to the routine of the service. After the application of the questionnaire, groups were formed for a period of one month in the three periods of the day, in order to cover several profiles of users and help in the construction of health knowledge and guidelines, also based on the Sanitary Reform Project and the daily life of the Sistema Único de Saúde (Unified Health System). The study was based on the Exploratory Research methodology, which aimed to provide greater privacy with the raised question, allowing the construction of hypotheses.

KEYWORDS: Health Promotion. Health centers. Urgency and Emergency. Social Work.

\section{INTRODUÇÃO}

A construção das políticas públicas no país vem de um processo longo e conturbado. $\mathrm{Na}$ atualidade, ainda lidamos cotidianamente com os reflexos dessa evolução diretamente nas práticas do Sistema Único de Saúde, comprometendo as ações de promoção em saúde.

Foi a partir da década de 80 que a Área da Saúde no Brasil contou com a participação de novos sujeitos na cena e na discussão política, inclusive de profissionais de saúde, levando para uma discussão ampliada sobre as condições de vida da população e sobre as propostas governamentais apresentadas, em tempos de democracia, período reconhecido como fundante da Reforma Sanitária, num processo que teve como marco institucional a $8^{\text {a }}$ Conferência Nacional de Saúde, realizada em 1986.

Serv. Soc. \& Saúde, Campinas, SP v.16, n. 1 (23), p. 25-48, jan./jun. 2017 E-ISSN 2446-5992 
Com a implantação do Sistema Único de Saúde, todos os cidadãos passaram a ter direito à saúde gratuita, instituído pela Constituição Federal de 1988, em seu artigo 196, como forma de efetivar o direito à saúde como direito de todos e dever do Estado e regulado pela Lei $n^{\circ}$ 8.080/1990. Sua operacionalização se dá através de diferentes níveis de atenção, de acordo com a complexidade das demandas e da intervenção necessária, processo inicialmente limitado, pois a formação dos profissionais ainda não era condizente com este novo modelo de promoção à saúde.

Como forma de adequar a ação profissional ao novo modelo de saúde foi usado o conceito preconizado na Carta de Ottawa, como resultado da discussão feita durante a Primeira Conferência Internacional sobre Promoção da Saúde, realizada em 1986.

Como resposta ao movimento que buscava renovar a concepção mundial de Saúde Pública foi possível estabelecer o consenso de que promover saúde significa fornecer à população condições para melhorar e exercer controle sobre sua própria saúde, aproveitando a vida de forma positiva, no sentido do uso de recursos pessoais e sociais, além de físicos. Busca-se assim, além da manutenção da "vida saudável", a obtenção de qualidade de vida.

Contudo, é imprescindível salientar que as ações para a qualidade e autonomia de vida apenas se tornam possíveis a partir de uma combinação de estratégias que incluam as ações do Estado, da comunidade, dos indivíduos e, principalmente, do Sistema de Saúde.

Pode-se entender que o adoecimento é uma manifestação singular e subjetiva, e é nesse momento que a proposta de grupos como promoção é fundamentada no conceito amplo de saúde, entendendo-o como processo de completo bem-estar, ampliando o olhar com vista à construção da autonomia dos sujeitos. Sendo assim, este tipo de trabalho possibilita a quebra da relação vertical, que tradicionalmente existe entre os profissionais da saúde e o usuário, como nos mostra Santos (2006).

A abordagem grupal tem sido usada como estratégia para atendimento em saúde desde o início do século 20, pois acaba por evidenciar a convivência com outras pessoas com igual condição clínica e exerce influência benéfica ao tratamento. Além disso, é recurso adequado para o desenvolvimento de ações de promoção da saúde, de acordo com Munari (2007). As práticas grupais no cotidiano da saúde coletiva, como ferramenta de promoção da 
saúde, integram a lista de reorientação dos serviços de saúde com ênfase na atenção básica proposta pelo Ministério da Saúde, por meio da Política Nacional de Promoção da Saúde.

Quando falamos em atendimento emergencial em saúde, estamos nos remetendo a um nível de complexidade específico dentro da hierarquização dos serviços de saúde e do Sistema Único de Saúde, expostos na Lei Orgânica da Saúde (LOS), assim como em diversos documentos que tratam sobre o assunto, como o Pacto Pela Saúde, A Política Nacional de Humanização e a Constituição Federal de 1988.

O sistema de saúde disposto de forma hierarquizada por níveis de complexidade e de forma regionalizada tem a tendência de permitir um conhecimento maior da situação de saúde da população de uma determinada área, o que favorece ações de Vigilância Epidemiológica, Sanitária, de Educação em Saúde, além das ações de atenção ambulatorial e hospitalar, de acordo com Souza (2001).

\section{O TRABALHO DO ASSISTENTE SOCIAL NA SALA DE ESPERA: PRIMEIRAS IMPRESSÕES}

Segundo Moreira e Pellizzaro (2009), é possível ver que a prática dos assistentes sociais vem se desenvolvendo e tornando-se uma prática necessária para a promoção e atenção à saúde. É uma discussão que vem se ampliando e ganhado forças frente à concepção de que o processo saúde-doença é determinado socialmente e reforçado pelo conceito de saúde.

A inserção do Serviço Social ocorre em todos os níveis de atenção à saúde e segundo Mioto e Nogueira os projetos societários em curso marcam essa atuação, com base no tripé: necessidades sociais em saúde, direito à saúde e produção da saúde.

As necessidades sociais em saúde são historicamente construídas e determinadas pelo movimento societário. $\mathrm{O}$ direito à saúde, mediado pelas políticas públicas, as quais refletem um patamar determinado da relação Estado e Sociedade, é operacionalizado através dos sistemas e serviços de saúde, envolvendo a gestão, o planejamento e a avaliação, além do controle social. A produção de saúde é entendida como um processo que se articula a partir das transformações econômicas, sociais e políticas, das ações de vigilância à saúde e das práticas de assistência à saúde (MIOTO; NOGUEIRA, 2009, p. 223). 
O trabalho dos assistentes sociais em um Serviço de Urgência e Emergência procura articular o exercício profissional com a constante pesquisa sobre a realidade, pois com esse conhecimento fica mais fácil compreender todas as transformações sociais postas pela contemporaneidade e, dessa forma trabalhar junto à população usuária as alternativas de enfrentamento.

A metodologia dos grupos em saúde, assim como as demais abordagens direcionadas à promoção da saúde, é constituída em torno de conceitos inter e transdisciplinares articulados especialmente nas últimas duas décadas. Portanto, a necessidade de pesquisas direcionadas à promoção, identificando possíveis intervenções, se coloca como um diferencial no desenvolvimento e aperfeiçoamento das tecnologias em saúde.

Foi realizado um trabalho/ação voltado à promoção para a emancipação possível do usuário, para que pudesse ser agente no processo de mudança de sua própria realidade. Por isso foi necessário conhecer a população durante a construção coletiva do grupo de sala de espera, percebendo-se o tempo escasso que a rotina impõe para as tarefas de cunho sócio educativo.

Foi necessário trabalhar com uma amostra em local previamente escolhido para verificar o possível surgimento de um ambiente crítico/reflexivo que possibilitou a acolhida aos usuários, levantando as suas necessidades e buscando intervir junto a eles e não apenas para eles, contribuindo para a efetivação dos princípios e diretrizes do Sistema Único de Saúde e a Política Nacional de Humanização.

Sendo assim, durante esta pesquisa, foi possível conhecer a identidade desse usuário, suas dificuldades e complexidades e suas necessidades, para confirmar a dificuldade de acesso e a falta de conhecimento do fluxo de saúde. A Unidade de Urgência e Emergência funciona durante 24 horas por dia, todos os dias da semana e sua equipe é formada por clínicos gerais, odontólogos, enfermeiros, técnicos de enfermagem, auxiliares de enfermagem, técnicos de radiologia, farmacêuticos, auxiliares de serviços gerais, assistentes sociais, psicólogos, assistentes administrativos, recepcionistas, coordenações, seguranças, equipes do SAMU (Serviço de Atendimento Móvel de Urgência) e equipes de Residência Multiprofissional (composta por profissionais de Enfermagem, Serviço Social, Farmácia, Odontologia e Psicologia). 
O fluxo consiste em acolhimento para classificação de risco (Protocolo Manchester), seguido de atendimento médico e das intervenções necessárias (exames, medicação, observação, alta ou transferência).

No contexto da UPH (Unidade Pré Hospitalar) referenciada como integrante de uma rede de saúde, é necessário entender a Rede de Atenção à Saúde (RAS) como uma proposta recente, tendo origem nas experiências de sistemas integrados de saúde, surgidas na primeira metade dos anos 90 nos Estados Unidos. A partir desta época, avançaram pelos sistemas públicos da Europa Ocidental e para o Canadá até atingir, posteriormente, alguns países em desenvolvimento.

Mendes (2011) apud Hartz e Contandriopoulos (2004) afirma que a integralidade da atenção é um eixo prioritário da investigação e avaliação dos serviços e dos Sistemas de Atenção à Saúde, estruturados como redes assistenciais interorganizacionais que articulam as dimensões clínicas, funcionais, normativas e sistêmicas em sua operacionalização, reconhecendo que nenhuma organização reúne a totalidade dos recursos e as competências necessárias para a solução dos problemas de saúde de uma população, em seus diversos ciclos de vida.

Torna-se, portanto, indispensável desenvolver mecanismos de cooperação e coordenação próprios de uma gestão eficiente e responsável dos recursos coletivos, que responda às necessidades de saúde individuais em âmbitos local e regional. Segundo Mendes (1998), isso se fará por meio de um "sistema sem muros", eliminando as barreiras de acesso entre os diversos níveis de atenção - do domicílio ao hospital, especialistas e generalistas, setores público e privado - ligados por corredores virtuais que ultrapassem, de fato, as fronteiras municipais com vistas à otimização de recursos e ampliação das oportunidades de cuidados adequados.

Esse movimento universal em busca da construção de RAS (Rede de Atenção à Saúde) está sustentado por evidências de que essas redes constituem uma saída para a crise contemporânea dos sistemas de atenção à saúde, provindas de vários países, constatando-se que melhoram os resultados sanitários e econômicos desses sistemas.

Sendo assim, é necessário que a Rede de Saúde seja articulada a fim de fornecer o atendimento integral e regionalizado, previsto na Política do SUS, assegurando aos usuários os serviços de referência e contra referência de qualidade e com efetividade.

Serv. Soc. \& Saúde, Campinas, SP v.16, n. 1 (23), p. 25-48, jan./jun. 2017 E-ISSN 2446-5992 


\section{MÉTODO E RESULTADOS}

A pesquisa foi realizada com 121 usuários que compunham o grupo, e apenas 60 usuários responderam ao questionário. Os encontros ocorreram duas vezes por semana, as terças e quintas-feiras, durante os três períodos (matutino, vespertino e noturno por 04 semanas, com duração - em média de 30 minutos - e com número mínimo de participantes de 03 usuários por encontro).

Neste primeiro momento, os pacientes estavam alocados na sala de medicação, onde se contava com poltronas para melhor acomodação. Foi preenchido um formulário para conhecimento sócio demográfico, abrangendo informações referentes à idade, gênero, estado civil, número de filhos, escolaridade, bairro e acesso a postos de saúde.

Para avaliar o nível de conhecimento dos usuários acerca do fluxo da unidade e da rede de saúde, foi aplicado um questionário com conteúdo simples, pontual e padronizado, construído pela pesquisadora e adaptado de acordo com a realidade da unidade e que buscou contemplar diversas realidades a qual o usuário é submetido, onde foi empregada a escala Likert, sendo acessível a usuários de todos os níveis de escolaridade, visando sua compreensão; composto por dez itens com cinco alternativas de respostas cada um, numa escala que variava entre muito satisfeito (cotação 1) e muito insatisfeito (cotação 5). A pontuação tem um valor mínimo de dez pontos e máximo de cinquenta pontos.

As tabelas abaixo foram feitas de acordo com a correlação e, por isso são apresentadas apenas as tabelas das análises que mostraram correlação com a questão correspondente.

Com base na Tabela 1, é somado o escore do questionário baseado na idade dos usuários participantes, sendo a idade mínima de 18 anos e máxima de 76 anos, sendo a média de 39,40 anos. A média total foi de 31,52, com desvio padrão de 3,90, mostrando nessa tabela que os usuários encontram-se de indiferentes a levemente insatisfeitos. $\mathrm{O}$ valor mínimo em relação ao escore total foi de 22 e o máximo de 39. 
Tabela 1. Análise descritiva das variáveis numéricas do questionário (n=60).

\begin{tabular}{c|c|c|c|c|c|c|c|c}
\hline Variável & $\mathbf{N}$ & Média & D.P & Mín. & Q1 & Q3 & Mediana & Máx. \\
\hline Idade & 60 & 39,40 & 15,75 & 18,00 & 27,50 & 49,00 & 35,50 & 76,00 \\
Questão 1 & 60 & 3,10 & 1,15 & 1,00 & 2,00 & 4,00 & 4,00 & 5,00 \\
Questão 2 & 60 & 2,87 & 1,10 & 1,00 & 2,00 & 4,00 & 2,00 & 5,00 \\
Questão 3 & 60 & 3,37 & 1,13 & 1,00 & 2,00 & 4,00 & 4,00 & 5,00 \\
Questão 4 & 60 & 3,70 & 0,93 & 1,00 & 4,00 & 4,00 & 4,00 & 5,00 \\
Questão 5 & 60 & 2,25 & 0,91 & 1,00 & 2,00 & 2,00 & 2,00 & 5,00 \\
Questão 6 & 60 & 2,70 & 1,20 & 1,00 & 2,00 & 4,00 & 2,00 & 5,00 \\
Questão 7 & 60 & 3,70 & 1,05 & 1,00 & 4,00 & 4,00 & 4,00 & 5,00 \\
Questão 8 & 60 & 3,35 & 1,09 & 1,00 & 2,00 & 4,00 & 4,00 & 5,00 \\
Questão 9 & 60 & 3,57 & 1,03 & 1,00 & 3,00 & 4,00 & 4,00 & 5,00 \\
Questão 10 & 60 & 2,92 & 1,08 & 1,00 & 2,00 & 4,00 & 3,00 & 5,00 \\
Escore Total & 60 & 31,52 & 3,90 & 22,00 & 29,00 & 34,00 & & 39,00 \\
\hline
\end{tabular}

FONTE: Dados próprios da pesquisa.

Tabela 2. Análise comparativa das variáveis categóricas entre gêneros.

\begin{tabular}{cccc}
\hline Questão 2 & \multicolumn{2}{c}{ Frequência e Percentual } & Total \\
& Feminino & Masculino & \\
\hline MS & 3 & 1 & 4 \\
& $7,69(\%)$ & $4,76(\%)$ & 27 \\
S & 21 & 6 & 3 \\
NR & $53,85(\%)$ & $28,57(\%)$ & 25 \\
& 0 & 3 & $14,29(\%)$ \\
I & $0,00(\%)$ & 10 & 1 \\
MI & 15 & $47,62(\%)$ & \\
& $38,46(\%)$ & 1 & 60 \\
\hline
\end{tabular}

Fonte: Dados próprios da pesquisa.

Serv. Soc. \& Saúde, Campinas, SP v.16, n. 1 (23), p. 25-48, jan./jun. 2017 E-ISSN 2446-5992 
Tabela 3. Análise comparativa das variáveis categóricas entre gêneros.

Questão 3

Feminino

Frequência e Percentual

Masculino

\begin{tabular}{|c|c|c|c|}
\hline MS & 3 & 0 & 3 \\
\hline & $7,69(\%)$ & $0,00(\%)$ & \\
\hline $\mathrm{S}$ & 9 & 5 & 14 \\
\hline & $23,08(\%)$ & $23,81(\%)$ & \\
\hline NR & $\begin{array}{c}2 \\
5,13(\%)\end{array}$ & $\begin{array}{c}7 \\
33,33(\%)\end{array}$ & 9 \\
\hline I & $\begin{array}{c}21 \\
53,85(\%)\end{array}$ & $\begin{array}{c}5 \\
23,81(\%)\end{array}$ & 26 \\
\hline MI & $\begin{array}{c}4 \\
10,26(\%)\end{array}$ & $\begin{array}{c}4 \\
19,05(\%)\end{array}$ & 8 \\
\hline & 39 & 21 & 60 \\
\hline
\end{tabular}

Total

Fonte: Dados próprios da pesquisa.

A Tabela 2 apresenta as comparações das variáveis entre os gêneros, que mostraram diferença significativa, nas seguintes questões: questão 2, maior frequência de NR $(14,29 \%)$ e I $(47,62 \%)$ no gênero masculino, e de S $(53,85 \%)$ no gênero feminino, demonstrando que os usuários do gênero masculino não frequentam a Unidade Básica de Saúde, enquanto as mulheres fazem uso do serviço. E na questão 3 (Tabela 3), maior frequência de NR $(33,33 \%)$ no sexo masculino, e de I $(53,85 \%)$ no sexo feminino, pois é referente a agilidade para marcação de consulta nas UBSs. Como o gênero feminino frequenta o serviço, acabam por ter o conhecimento dos entraves referentes a este serviço.

Tabela 4. Análise comparativa das variáveis categóricas entre Estado Civil.

\begin{tabular}{cccc}
\hline Questão 1 & \multicolumn{2}{c}{ Frequência e Percentual } & Total \\
\hline MS & Com companheiro & Sem companheiro & 3 \\
S & 1 & 2 & \\
& $3,85(\%)$ & $5,88(\%)$ & 24 \\
NR & 7 & 17 & 30 \\
I & $26,92(\%)$ & $50,00(\%)$ & 3 \\
& 0 & 0 & \\
MI & $0,00(\%)$ & $0(\%)$ & 60 \\
& 18 & 12 & \\
Total & $69,23(\%)$ & $35,29(\%)$ & 60 \\
\hline
\end{tabular}

FONTE: Dados próprios da pesquisa.

Serv. Soc. \& Saúde, Campinas, SP v.16, n. 1 (23), p. 25-48, jan./jun. 2017 E-ISSN 2446-5992 
A Tabela 4 apresenta as comparações das variáveis categóricas entre o estado civil agrupados em "sem companheiro" (solteiro, divorciado e viúvo) e "com companheiro" (casado), para maior consistência nas análises.

Pelos resultados, verificou-se diferença significativa entre estado civil para a questão 1, maior frequência de I nos com companheiro $(69,23 \%)$ e de $S$ nos sem companheiro (50,00\%). Ou seja, segundo as variáveis é possível colocar que os usuários com companheiro sentem que conhecem menos a rede de saúde, enquanto que os usuários que não possuem companheiro consideram seu conhecimento satisfatório.

Tabela 5. Análise comparativa das variáveis categóricas entre Número de Filhos.

\begin{tabular}{|c|c|c|c|c|}
\hline \multirow[t]{2}{*}{ Questão 6} & \multicolumn{2}{|c|}{ Frequência e Percentual } & \multirow[b]{2}{*}{$>=3$} & \multirow[t]{2}{*}{ Total } \\
\hline & 0 & $1-2$ & & \\
\hline \multirow[t]{2}{*}{ MS } & 2 & 0 & 4 & 6 \\
\hline & $14,29(\%)$ & $0,00(\%)$ & $25,00(\%)$ & \\
\hline \multirow[t]{2}{*}{$\mathrm{S}$} & 4 & 17 & 10 & 31 \\
\hline & $28,57(\%)$ & $56,67(\%)$ & $62,50(\%)$ & \\
\hline \multirow[t]{2}{*}{ NR } & 1 & 2 & 0 & 3 \\
\hline & $7,14(\%)$ & $6,67(\%)$ & $0,00(\%)$ & \\
\hline \multirow[t]{2}{*}{ I } & 6 & 7 & 2 & 15 \\
\hline & $42,86(\%)$ & $23,33(\%)$ & $12,50(\%)$ & \\
\hline \multirow[t]{2}{*}{ MI } & 1 & 4 & 0 & 5 \\
\hline & $7,14(\%)$ & $13,33(\%)$ & $0,00(\%)$ & \\
\hline Total & 14 & 30 & 16 & 60 \\
\hline
\end{tabular}

FONTE: Dados próprios da pesquisa.

A Tabela 5 apresenta as comparações das variáveis categóricas entre o número de filhos. Pelos resultados, verificou-se diferença significativa entre número de filhos para a questão 6, maior frequência de I nos sem filhos (42,86\%) e de S nos com 1-2 (56,67\%) ou $\geq 3$ filhos (62,50\%). Portanto, é considerado que os usuários que não possuem filhos consideram a agilidade para o atendimento na Unidade Pré-Hospitalar como insatisfatória e os usuários que possuem (a partir de) 1 filho consideram satisfatória a rapidez para atendimento nesta mesma unidade. 
Tabela 6. Análise comparativa das variáveis categóricas entre Escolaridade.

\begin{tabular}{|c|c|c|c|c|}
\hline \multirow[t]{2}{*}{ Questão 3} & \multicolumn{2}{|c|}{ Frequência e Percentual } & \multirow[b]{2}{*}{$3^{\circ} \mathrm{Grau}$} & \multirow[t]{2}{*}{ Total } \\
\hline & $1^{\circ} \mathrm{Grau}$ & $2^{\circ} \mathrm{Grau}$ & & \\
\hline \multirow[t]{2}{*}{ MS } & 2 & 0 & 1 & 3 \\
\hline & $9,09(\%)$ & $0,00(\%)$ & $7,69(\%)$ & \\
\hline \multirow[t]{2}{*}{$S$} & 6 & 8 & 0 & 14 \\
\hline & $27,27(\%)$ & $32,00(\%)$ & $0,00(\%)$ & \\
\hline \multirow[t]{2}{*}{ NR } & 0 & 5 & 4 & 9 \\
\hline & $0,00(\%)$ & $20,00(\%)$ & $30,77(\%)$ & \\
\hline \multirow[t]{2}{*}{ I } & 10 & 11 & 5 & 26 \\
\hline & $45,45(\%)$ & $44,00(\%)$ & $38,46(\%)$ & \\
\hline \multirow[t]{2}{*}{ MI } & 4 & 1 & 3 & 8 \\
\hline & $18,18(\%)$ & $4,00(\%)$ & $23,08(\%)$ & \\
\hline Total & 22 & 25 & 13 & 60 \\
\hline \multirow[t]{2}{*}{ Questão 6 } & \multicolumn{2}{|c|}{ Frequência e Percentual } & & Total \\
\hline & $1^{\circ} \mathrm{Grau}$ & $2^{\circ} \mathrm{Grau}$ & $3^{\circ} \mathrm{Grau}$ & \\
\hline \multirow[t]{2}{*}{ MS } & 4 & 0 & 2 & 6 \\
\hline & $18,18(\%)$ & $0,00(\%)$ & $15,38(\%)$ & \\
\hline \multirow[t]{2}{*}{ S } & 10 & 15 & 6 & 31 \\
\hline & $45,45(\%)$ & $60,00(\%)$ & $46,15(\%)$ & \\
\hline \multirow[t]{2}{*}{ NR } & 1 & 0 & 2 & 3 \\
\hline & $4,55(\%)$ & $0,00(\%)$ & $30,77(\%)$ & \\
\hline \multirow[t]{2}{*}{ I } & 7 & 5 & 3 & 15 \\
\hline & $31,82(\%)$ & $20,00(\%)$ & $23,08(\%)$ & \\
\hline \multirow[t]{2}{*}{ MI } & 0 & 5 & 0 & 5 \\
\hline & $0(\%)$ & $20,00(\%)$ & $0,00(\%)$ & \\
\hline Total & 22 & 25 & 13 & 60 \\
\hline \multirow[t]{2}{*}{ Questão 7} & \multicolumn{2}{|c|}{ Frequência e Percentual } & & Total \\
\hline & $1^{\circ} \mathrm{Grau}$ & $2^{\circ} \mathrm{Grau}$ & $3^{\circ} \mathrm{Grau}$ & \\
\hline \multirow[t]{2}{*}{ MS } & 2 & 0 & 0 & 02 \\
\hline & $9,09(\%)$ & $0,00(\%)$ & $0,00(\%)$ & \\
\hline \multirow[t]{2}{*}{$\mathrm{S}$} & 3 & 4 & 3 & 10 \\
\hline & $13,64(\%)$ & $16,00(\%)$ & $23,08(\%)$ & \\
\hline \multirow[t]{2}{*}{ NR } & 0 & 0 & 2 & 2 \\
\hline & $0,00(\%)$ & $0,00(\%)$ & $15,38(\%)$ & \\
\hline \multirow[t]{2}{*}{ I } & 10 & 20 & 6 & 36 \\
\hline & $45,45(\%)$ & $80,00(\%)$ & $46,15(\%)$ & \\
\hline \multirow[t]{2}{*}{ MI } & 7 & 1 & 2 & 10 \\
\hline & $31,82(\%)$ & $4,00(\%)$ & $15,38(\%)$ & \\
\hline Total & 22 & 25 & 13 & 60 \\
\hline
\end{tabular}

Serv. Soc. \& Saúde, Campinas, SP v.16, n. 1 (23), p. 25-48, jan./jun. 2017 E-ISSN 2446-5992 


\begin{tabular}{|c|c|c|c|c|}
\hline \multirow[t]{2}{*}{ Questão 8} & \multicolumn{2}{|c|}{ Frequência e Percentual } & \multirow[b]{2}{*}{$3^{\circ} \mathrm{Grau}$} & \multirow[t]{2}{*}{ Total } \\
\hline & $1^{\circ} \mathrm{Grau}$ & $2^{\circ} \mathrm{Grau}$ & & \\
\hline MS & $\begin{array}{c}1 \\
4,55(\%)\end{array}$ & $\begin{array}{c}0 \\
0,00(\%)\end{array}$ & $\begin{array}{c}0 \\
0.00(\%)\end{array}$ & 1 \\
\hline$S$ & $\begin{array}{c}6 \\
27,27(\%)\end{array}$ & $\begin{array}{c}7 \\
28,00(\%)\end{array}$ & $\begin{array}{c}6 \\
46,15(\%)\end{array}$ & 19 \\
\hline NR & $\begin{array}{c}2 \\
9,09(\%)\end{array}$ & $\begin{array}{c}0 \\
0,00(\%)\end{array}$ & $\begin{array}{c}2 \\
15,38(\%)\end{array}$ & 4 \\
\hline I & $\begin{array}{c}8 \\
36,36(\%)\end{array}$ & $\begin{array}{c}18 \\
72,00(\%)\end{array}$ & $\begin{array}{c}4 \\
30,77(\%)\end{array}$ & 30 \\
\hline MI & $\begin{array}{c}5 \\
22,73(\%)\end{array}$ & $\begin{array}{c}0 \\
0,00(\%)\end{array}$ & $\begin{array}{c}1 \\
7,69(\%)\end{array}$ & 6 \\
\hline Total & 22 & 25 & 13 & 60 \\
\hline Questão 9 & \multicolumn{2}{|c|}{ Frequência e Percentual } & $3^{\circ} \mathrm{Grau}$ & Total \\
\hline MS & $\begin{array}{c}1 \\
4,55(\%)\end{array}$ & $\begin{array}{c}0 \\
0,00(\%)\end{array}$ & $\begin{array}{c}0 \\
0,00(\%)\end{array}$ & 1 \\
\hline$S$ & $\begin{array}{c}7 \\
31,82(\%)\end{array}$ & $\begin{array}{c}5 \\
20,00(\%)\end{array}$ & $\begin{array}{c}1 \\
7,69(\%)\end{array}$ & 13 \\
\hline NR & $\begin{array}{c}2 \\
9,09(\%)\end{array}$ & $\begin{array}{c}1 \\
4,00(\%)\end{array}$ & $\begin{array}{c}2 \\
15,38(\%)\end{array}$ & 5 \\
\hline I & $\begin{array}{c}7 \\
31,82(\%)\end{array}$ & $\begin{array}{c}19 \\
76,00(\%)\end{array}$ & $\begin{array}{c}7 \\
53,85(\%)\end{array}$ & 33 \\
\hline MI & $\begin{array}{c}5 \\
22,73(\%)\end{array}$ & $\begin{array}{c}0 \\
0,00(\%)\end{array}$ & $\begin{array}{c}3 \\
23,08(\%)\end{array}$ & 8 \\
\hline Total & 22 & 25 & 13 & 60 \\
\hline
\end{tabular}

FONTE: Dados próprios da pesquisa.

A Tabela 6 apresenta as comparações das variáveis categóricas entre os níveis de escolaridade. Pelos resultados, verificou-se diferença significativa entre escolaridade para a questão 3 (teste exato de Fisher: p=0,013), onde ocorre maior frequência de $\mathrm{S}$ nos com $1^{\circ}$ grau $(27,27 \%)$ e $2^{\circ}$ grau $(32,00 \%)$, e de NR $(30,77 \%)$ e MI $(23,08 \%)$ nos com $3^{\circ}$ grau. O que representa que os usuários com Primeiro e Segundo (completo e incompleto) sentem-se mais satisfeitos com a agilidade de marcação de consultas na UBS, enquanto os usuários com Terceiro Grau (completo ou incompleto) se dizem indiferentes e/ou muito insatisfeitos.

Na questão 6 (segundo teste exato de Fisher: $\mathrm{p}=0,025$ ) há maior frequência de I nos com $1^{\circ}$ grau $(31,82 \%)$, e de $S(60,00 \%)$ e MI $(20,00 \%)$ nos com $2^{\circ}$ grau. O que faz alusão à insatisfação no atendimento na UPH (demora no atendimento) para os usuários com Primeiro Grau (completo ou incompleto), não souberam responder e/ou se mostraram indiferentes os Serv. Soc. \& Saúde, Campinas, SP v.16, n. 1 (23), p. 25-48, jan./jun. 2017 E-ISSN 2446-5992 
usuários com Segundo Grau (completo ou incompleto) e muito insatisfeito os usuários com Terceiro Grau/Ensino Superior (completo ou incompleto).

Na questão 7 ocorre maior frequência de MI nos com $1^{\circ}$ grau $(31,82 \%)$, e de I nos com $2^{\circ}$ grau $(80,00)$. Por se tratar da orientação referente à Classificação de Risco o estudo mostra que os usuários de Primeiro e Segundo Grau (completo ou incompleto) estão insatisfeitos e muito insatisfeitos com a falta de orientação sobre o tema.

Na questão 8 há maior frequência de MI nos com $1^{\circ}$ grau $(22,73 \%)$, de I nos com $2^{\circ}$ grau $(72,00 \%)$, e de $\mathrm{S}$ nos com $3^{\circ}$ grau $(46,15 \%)$. Esta questão aborda o entendimento e a anuência sobre a classificação de risco e o fluxo da unidade. Percebe-se então, com o estudo, que quanto menor o grau de escolaridade, menor o entendimento sobre a forma de funcionamento da UPH.

Por fim, a questão 9 demonstrou maior frequência de $\mathrm{S}$ nos com $1^{\circ}$ grau $(31,82 \%)$, e de I nos com $2^{\circ}$ grau $(76,00 \%)$. Esta questão discorre sobre a participação em grupos que discutem a saúde (conselhos municipais, grupos nas unidades e etc.) e que, no caso do usuário não participar mensura o desejo de envolver-se com o tema. A análise traz que os usuários em número expressivo, com o Primeiro Grau (completo ou incompleto) não participam de nenhum grupo e não possuem o desejo. Os usuários com o Segundo Grau mostram-se insatisfeitos com a própria não-participação e colocam que se houvesse tempo hábil em sua rotina, participariam dos grupos.

\section{DISCUSSÃO}

A situação dos serviços de emergência é, hoje, motivo de preocupação para a comunidade sanitária e a sociedade em geral. Um dos argumentos apresentados para a demanda excessiva nos serviços de urgência/emergência hospitalar é que a maior parte dos atendimentos realizados é decorrente de problemas "simples" que poderiam ser resolvidos em Serviços de Atenção Básica ou especializados ou, ainda, em serviços de urgência de menor complexidade. Esse perfil de demanda configura uma das principais portas de entrada ao sistema de saúde e possivelmente revela, entre outras coisas, distribuição desigual da oferta de serviços, não apenas do ponto de vista quantitativo, mas, também, qualitativo, na Atenção Básica, especializada e também na hospitalar.

Serv. Soc. \& Saúde, Campinas, SP v.16, n. 1 (23), p. 25-48, jan./jun. 2017 E-ISSN 2446-5992 
A intervenção nos mostra que é possível a percepção de que este resultado não implica na impossibilidade de realização de um grupo em promoção de saúde na Unidade de Urgência e Emergência. Deve-se levar em consideração que, atualmente, o que falta é estratégia para atingir essa população e meios de atingir o potencial pensado inicialmente, pois o estudo conceitual indica que a realização de grupos na saúde coletiva pode estar se consolidando como uma estratégia de dimensão educativa-participativa e de empoderamento dos sujeitos na comunidade em que estão inseridos.

Através das informações obtidas por meio do questionário sócio demográfico foi possível perceber que há um percentual expressivo de mulheres que frequentam as Unidades de Saúde (65,00\%); este é um dado já apontado em outras pesquisas, que concluem que mesmo diante da elevada taxa de mortalidade masculina, os homens procuram menos os serviços de saúde.

Entretanto, apesar de as taxas masculinas assumirem um peso significativo nos perfis de morbimortalidade, observa-se que a presença de homens nos serviços de Atenção Básica à saúde é menor do que a das mulheres (GOMES et al, 2007; COURTENAY, 2000).

Há a hipótese de diversos fatores, tais como: paradigmas sociais, culturais e gênero, o que prejudica diretamente a prevenção e promoção em saúde, pois a procura feminina pelos serviços de saúde é associada à fraqueza e feminilidade, reflexos de uma sociedade com perfil patriarcal. Além de se considerar que majoritariamente, os homens não tem conhecimento dos serviços oferecidos pela Rede de Saúde (PEREIRA; NERY, 2014).

É de nosso conhecimento que, em grande maioria, as Unidades Básicas de Saúde funcionam de segunda a sexta em horário comercial, o que torna seus horários praticamente incompatíveis com a disponibilidade do homem que trabalha. No entanto, sabe-se que muitas mulheres que utilizam os serviços de saúde também trabalham fora de casa o que não justificaria a "desculpa" utilizada pela maioria dos homens.

Dessa forma, como sugere Machin et al, (2011), o trabalho da mulher, mesmo aquele remunerado, não é visto como uma forma socialmente justificada para explicar uma possível indisponibilidade para o serviço de saúde. O tempo feminino parece ser visto como o que está a serviço dos cuidados em saúde. Neste sentido, Schraiber (2005), destaca que a flexibilização do horário de trabalho das mulheres para frequentar os serviços indica haver, por parte dos empregadores, a presença do imaginário social de que a mulher tem que se Serv. Soc. \& Saúde, Campinas, SP v.16, n. 1 (23), p. 25-48, jan./jun. 2017 E-ISSN 2446-5992 
cuidar.

Em consonância com este dado, podemos refletir e, possivelmente, fundamentar a correlação estatística obtida entre insatisfação e gênero, uma vez as mulheres se encontraram mais insatisfeitas frente ao atendimento na UBS e a demora no agendamento de consultas. Por serem usuárias diretas do serviço e estarem presentes no território, há um maior conhecimento acerca das debilidades de uma unidade.

Partindo dessa ideia, em março de 2008, com o objetivo de complementar a Atenção Básica, o Ministério da Saúde lançou uma proposta de Política de Atenção à Saúde do Homem, a Política Nacional de Atenção Integral à Saúde do Homem (PNAISH), a qual visa nortear as ações de atenção integral à saúde do homem, buscando estimular o autocuidado, principalmente, o reconhecimento por parte do homem que a saúde é um direito de cidadania de todos os indivíduos do gênero masculino.

Na Unidade de Urgência e Emergência ocorre o mesmo, dado que nos faz refletir, uma vez que a UPH é uma unidade 24 horas. Leva-se em consideração que o estudo foi realizado em sala separada, após o acolhimento. É perceptível na unidade que no Setor de Observação (onde aguardam os usuários que necessitam de internação) há maior incidência do sexo masculino, embora não se tenha estudos validados com essa informação.

Contextualizo que as Unidades Básicas de Saúde deveriam funcionar como portas de entrada ao sistema, oferecendo resolutividade e assistência integral. No entanto, na prática, isso não ocorre e a porta de entrada tem sido as Unidades de Pronto Atendimento (BATISTELA, 2008 apud CECILIO, 1997).

Com estes dados, é perceptível a necessidade de que os profissionais da área da saúde da Rede Básica devam agir em conjunto, efetivando o ideal de uma "rede de saúde" articulada. Devem ser realizadas ações em conjunto com vistas a acolher os usuários de forma a criar vínculo entre ambas as partes, além de garantir resolutividade nas ações o que acaba por gerar mais confiança e, posteriormente, maior frequência da população nas UBSs.

Consequentemente à falta de vínculo com a UBS, mostra-se uma alta porcentagem referente à demanda nos serviços de urgência e emergência e observa-se um fluxo desordenado dos usuários nestes serviços, o que faz necessária a reorganização do processo de trabalho ponderando o atendimento aos diferentes graus de especificidade (prioridades) e Serv. Soc. \& Saúde, Campinas, SP v.16, n. 1 (23), p. 25-48, jan./jun. 2017 E-ISSN 2446-5992 
não por ordem de chegada considerando a Classificação de Risco de acordo com o Protocolo Manchester (BRASIL, 2004).

Sendo assim, também é necessário que seja claro para todos (trabalhadores e usuários do SUS), o fluxograma municipal de referência e contra referência para acesso aos serviços de saúde, bem como a orientação do fluxo interno da unidade e a Classificação de Risco a fim de entender e cooperar com o fluxo estabelecido pela unidade.

Essa orientação deve ser feita no momento do acolhimento, entendendo-o como ação humanizadora e acolhedora, não se restringindo ao espaço físico, mas sim a toda situação e em todos os momentos de atendimento praticado pelos trabalhadores de saúde (MATUMOTO, 1998), e deve ser entendido como uma ferramenta de intervenção na qualidade da escuta, construção de vínculo, garantia do acesso com responsabilização e resolutividade nos serviços.

O estudo mostra que os usuários, atualmente, não são orientados acerca desses temas. De acordo com as respostas, 76,67\% dos usuários não são orientados sobre a Classificação de Risco em nenhum momento durante sua passagem pela unidade (desde a abertura da ficha até a conclusão da mesma). E 60,00\% não entende, e por isso, não concorda, com o fluxo proposto pela unidade.

Além disso, os dados nos mostraram que outros dois fatores podem repercutir na participação dos usuários na Rede de Saúde: o fato de estar casado associado à presença de filhos, pois essa condição pode ocasionar um acúmulo de tarefas, desfavorecendo a qualidade no acesso, devido aos cuidados dispensados aos filhos e a família.

A mulher de hoje multiplicou funções, mas a sociedade ainda não dividiu as responsabilidades. A divisão de tarefas domiciliares não ocorre de maneira igualitária entre os membros familiares, o que gera sobrecarga para as mulheres. Ou seja, o papel feminino mudou, enquanto que o papel masculino quase não foi modificado. A sociedade atual exige, e a própria mulher acaba exigindo de si mesma, que ela seja múltipla (ROCHA-COUTINHO, 2004).

Segundo os dados colhidos e presentes na Tabela 3, há maior frequência de insatisfação nos usuários com companheiro $(69,23 \%)$, ou seja, há menor conhecimento da rede de saúde, o que corrobora com o perfil da mulher esperado pela sociedade, que ela seja 
múltipla, mas caso tenha que realizar uma opção, a escolha considerada correta do ponto de vista patriarcal, é que ela satisfaça as necessidades domésticas. No entanto, referente à agilidade no atendimento na UPH há maior frequência de insatisfação com a possível demora nos usuários sem filhos $(50,00 \%)$ e satisfação com o tempo em espera pelos usuários com mais de 3 filhos $(62,50 \%)$.

Por fim, o estudo mostrou que se deve refletir sobre os diferentes graus de escolaridade e as implicações na saúde, conforme a Tabela 5 nos mostra ao realizar as correlações. No que se refere à Unidade Básica de Saúde, os usuários com Primeiro Grau e Segundo Grau (completo ou incompleto) mostram-se satisfeitos com a rotina da UBS, enquanto os usuários com o Terceiro Grau (completo ou incompleto) mostram-se insatisfeitos com a demora para marcação de consultas de rotina.

Em comparação com a questão 6 , ocorre maior insatisfação com a demora no atendimento na UPH nos usuários com Primeiro Grau (completo e incompleto), o que pode ser entendido que o usuário não tem acesso as informações de fluxograma da UPH, o que dificulta seu entendimento e aumenta sua insatisfação.

Essa afirmação é perceptível na questão 7, onde ocorre maior frequência de MI nos com $1^{\circ}$ grau $(31,82 \%)$, e de I nos com $2^{\circ}$ grau $(80,00)$. Essa questão se refere à falta de orientação a respeito da Classificação de Risco. Em relação a assertiva de entendimento e anuência sobre a Classificação de Risco e Fluxo da Unidade, colocada na questão 8 há maior insatisfação nos usuários de Primeiro e Segundo Grau (completo ou incompleto), enquanto ocorre satisfação nos usuários com Terceiro Grau, ou seja, há um entendimento maior conforme aumenta o nível de escolaridade.

Segundo Nascimento (2014), a baixa escolaridade é uma realidade em muitos municípios do país e esse é um indicador que influencia de forma negativa a conscientização de que cada usuário é um agente promotor de saúde para si mesmo e para a coletividade. Deve-se concluir que a equipe de saúde pode ser promotora de educação como instituição formada por cidadãos e interessada na melhoria da qualidade de vida da população que assiste.

Ademais, o estudo "Estratégias voltadas para o autocuidado do paciente", realizado em Uberaba/MG, em 2014, nos mostra que há falta de entendimento e falta de adesão a Serv. Soc. \& Saúde, Campinas, SP v.16, n. 1 (23), p. 25-48, jan./jun. 2017 E-ISSN 2446-5992 
tratamentos por pacientes com baixo nível de escolaridade, e o "nó critico deste problema é o nível de informação da população e a falta de orientação adequada dos membros da equipe de saúde" (ALMEIDA, 2014). O estudo ainda traz que o investimento em estratégias voltadas para o autocuidado é uma medida que tende a aumentar a resolutividade, diminuindo os custos voltados para a Atenção Secundária, pois ocorre uma intervenção que auxilia na conscientização da identidade social.

Além disso, ao analisar a questão 9 percebemos a paridade com as questões anteriores, pois esta questão mostra que não há participação e o desejo em participar de grupos que discutem as questões e ações de saúde nos usuários com o Primeiro Grau $(36,37 \%)$ e Segundo Grau (20,00\%). E ocorre nos usuários de Segundo Grau $(76,00)$ e Terceiro Grau $(53,85)$ a falta de participação em grupos com esse viés, porem há o desejo.

No tocante a esse último dado, é colocado que falta tempo dentro dos afazeres para participar de forma efetiva de discussões sobre a política de saúde. Há também a relevância de que muitos usuários não entendem esses espaços como populares, discussão que foi realizada no grupo após a aplicação do questionário.

Foi possível perceber que é escassa a participação dos usuários nas ações de saúde e não há o conhecimento que essa participação é parte da Educação Popular em Saúde que está prevista como política do SUS com a perspectiva de tornar a saúde mais humanizada e em sintonia com a cultura popular. Conforme Vasconcelos (2004), a Educação Popular é um saber indispensável para o estabelecimento da participação, e serve tanto para a formação da consciência sanitária como para a democratização radical das políticas públicas. Inclui um estilo de comunicação e ensino e um instrumento de gestão participativa da ação social, como prática horizontal.

É importante ressaltar ainda, que o conceito de educação em saúde está ancorado no conceito de promoção da saúde, que trata de processos que abrangem a participação de toda a população no contexto de sua vida cotidiana e não apenas das pessoas sob-risco de adoecer (LOPES et al, 2007).

Segundo o CFESS (2010), "cabe ao assistente social potencializar a orientação social com vistas à ampliação do acesso dos indivíduos e da coletividade aos direitos sociais" e também, "sensibilizar os usuários acerca dos direitos sociais, princípios e diretrizes do SUS, rotinas institucionais, promoção da saúde e prevenção de doenças por meio de grupos Serv. Soc. \& Saúde, Campinas, SP v.16, n. 1 (23), p. 25-48, jan./jun. 2017 E-ISSN 2446-5992 
socioeducativos". Este processo deve antepor a atenção coletiva e em grupo, pois possibilita a troca de experiências e ocorre a manifestação da força que os usuários tem. Para tanto, segundo Martins (2003) grupos auxiliam neste atendimento, pois criam uma unidade de indivíduos que interagem entre si e compartilham objetivos.

O grupo de sala de espera foi implementado como forma de atender às necessidades da população com o intuito de amenizar o desgaste físico e emocional associado ao tempo de espera para o atendimento, colocando-se como facilitador na troca de saberes entre participantes e profissionais presentes na sala de espera. É uma forma de abrir espaços de diálogo com a população, gerando possibilidades de reflexão e problematização, que favoreçam estratégias mais efetivas e humanas no processo de trabalho em saúde, para os usuários e, consequentemente, para os profissionais.

\section{CONSIDERAÇÕES FINAIS}

Durante a intervenção tivemos a oportunidade de entender os instrumentais da profissão, as bases do Sistema Único de Saúde, o trabalho de referência e contra referência, intersetorialidade e aliar o conhecimento com a convivência e enfrentamento das diferentes demandas ao longo do projeto.

Entendemos que o grupo de promoção à saúde é uma ferramenta adequada ao sistema de saúde, partindo do pressuposto de significar um processo de capacitação da comunidade em busca de melhores condições de vida e saúde com uma combinação de ações do Estado, da comunidade - reforçando ações locais e por fim, dos indivíduos, desenvolvendo habilidades que devem ser estimuladas com reflexão e ampliação da capacidade de entender a própria realidade e enfrentá-la.

Com este foco da saúde consideramos, além da participação dos indivíduos na melhoria das condições de vida, as parcerias intersetoriais como forma de aprimorar as estruturas necessárias, como maior vinculação entre os serviços do próprio território.

Entendendo assim, a intersetorialidade como uma relação horizontal entre os profissionais de diferentes formações convergindo para os mesmos objetivos e utilizando o grupo, que não deve ser realizado apenas com o profissional do Serviço Social, para ações mobilizadoras, pensando na real necessidade dos usuários do município.

Serv. Soc. \& Saúde, Campinas, SP v.16, n. 1 (23), p. 25-48, jan./jun. 2017 E-ISSN 2446-5992 
A prática do assistente social deve vir ao encontro com outros profissionais, auxiliando a unidade a buscar um embasamento suficiente acerca do sistema de saúde para realizar uma construção conjunta, onde há benefícios para todos os envolvidos no processo (usuários, comunidade, território, trabalhadores e gestão).

Por conseguinte, entendemos que mesmo com os obstáculos encontrados diariamente em função das condições precárias do sistema de saúde no país, cabe ao profissional superálos como forma de atingir o objetivo de desenvolver um trabalho que promova o crescimento da população. Esta ação deve ser direcionada como forma de organizar a intervenção grupal, como fruto do conhecimento, com bases consistentes e com criticidade, utilizando o grupo como ferramenta de assistência na saúde.

Recebido em 12.02.2017 - Aprovado em 28.06.2017

\section{REFERÊNCIAS}

ALMEIDA, A. C. V. Estratégias voltadas para o autocuidado do paciente. Universidade Federal do Triângulo Mineiro. Uberaba, 2014. Monografia (Especialização em Atenção Básica em Saúde da Família). Disponível em: http://www.nescon.medicina.ufmg.br/biblioteca/imagem/estrategias-voltadasautocuidado.pdf. Acesso em: 01 de Dezembro de 2016.

BATISTELA, S. et al. Os motivos de procura pelo Pronto Socorro Pediátrico de um Hospital Universitário referidos pelos pais ou responsáveis. Semina: Ciências Biológicas e da Saúde, Londrina, v. 29, n. 2, p. 121-130, jul./dez. 2008. Disponível em:

http://www.uel.br/proppg/portal/pages/arquivos/pesquisa/semina/pdf/semina_29_2_20_28.pd f. Acesso em: 10 de Dezembro de 2016.

BRASIL (1990). Diário Oficial da União. Lei nº 8080/90. Dispõe sobre as condições para promoção, proteção e recuperação da saúde, a organização e o financiamento dos serviços correspondentes e da outras providências. Brasília, DF.

BRASIL. Ministério da Saúde. Política Nacional de Humanização. Disponível em: <www.saude.gov.br>. Acesso em: 28 de maio de 2015.

BRASIL. MEC. Resolução no 15, de 13 de março de 2002, Diretrizes Curriculares do Curso de Serviço Social.

Serv. Soc. \& Saúde, Campinas, SP v.16, n. 1 (23), p. 25-48, jan./jun. 2017 E-ISSN 2446-5992 
BRASIL. Ministério da Saúde. Acolhimento com avaliação e classificação de risco. Brasília, 2004.

BRASIL. Ministério da Saúde. Secretaria Executiva. Núcleo Técnico da política Nacional de Humanização. HumanizaSUS: Acolhimento com avaliação e classificação de risco: um paradigma ético-estético no fazer em saúde. Brasília; 2004

BRASIL. Ministério da Saúde. Secretaria de Atenção à Saúde. Departamento de Ações Programáticas Estratégicas. Política Nacional de Atenção Integral à Saúde do Homem. Brasília: 2009.

CONSELHO Federal de Serviço Social. Parâmetros para atuação de assistentes sociais na política de saúde. Brasília: CFESS, 2010.

COURTENAY, W.H. Construction of masculinity and their influence on men's wellbeing: a theory of gender and health. Social Science \& Medicine, vol. 50, Issue 10, p. 1385- 1401, 2000 .

GOMES, R.; NASCIMENTO, E. F. do; ARAUJO, F. C. de. Por que os homens buscam menos os serviços de saúde do que as mulheres? As explicações de homens com baixa escolaridade e homens com ensino superior. Cadernos de Saúde Pública, Rio de Janeiro, v. 23, n. 3, Mar. 2007. Acesso em: 12 de Novembro de 2016.

HARTZ, Z. M. de A. ; CONTANDRIOPOULOS, A. - Integralidade da atenção e integração de serviços: desafios para avaliar um sistema "sem muros". Cadernos de Saúde Pública, 20: 5331-5336, 2004.

INSTITUTO BRASILEIRO DE GEOGRAFIA E ESTATÍSTICA - IBGE. Sinopse do Senso Demográfico de 2010. Rio de Janeiro, 2011.

LOPES, E. F. et al. Educação em Saúde: Um desafio para a transformação da práxis no cuidado em Enfermagem. Revista HCPA, v. 2, n. 27, p. 25-17, 2007. Disponível em: <http://www.seer.ufrgs.br/hcpa/article/viewFile/2063/1178>. Acesso em: 23 de maio de 2015.

MACHIN, R. et al. Concepções de gênero, masculinidade e cuidados em saúde: estudo com profissionais de saúde da atenção primária. Ciência e Saúde Coletiva, Rio de Janeiro, v. 16, n. 11, Nov. 2011.

MARTINS, S. T. F. Processo grupal e a questão do poder em Martín-Baró. Psicologia e Sociedade. Belo Horizonte, v. 15, n. 1, Jan. 2003. Disponível em:

<http://www.scielo.br/pdf/psoc/v15n1/v15n1a11.pdf >. Acesso em: 15 Outubro de 2015. Serv. Soc. \& Saúde, Campinas, SP v.16, n. 1 (23), p. 25-48, jan./jun. 2017 E-ISSN 2446-5992 
MATUMOTO, S. O acolhimento: um estudo sobre os seus componentes e sua produção em uma Unidade da Rede Básica de Serviços de Saúde. 1998. Dissertação (Mestrado) - Escola de Enfermagem de Ribeirão Preto, Universidade de São Paulo, Ribeirão Preto, 1998. Disponível em: http://www.teses.usp.br/teses/disponiveis/22/22133/tde-14012009164720/en.php. Acesso em: 30 de setembro de 2016.

MENDES, E. V. - A reengenharia do sistema de serviços de saúde no nível local: a gestão da atenção à saúde. In: MENDES, E. V. (Org.) A organização da saúde no nível local. São Paulo, Ed. HUCITEC, 1998.

MENDES, E. V. As Redes de Atenção à Saúde. 2a . ed. Brasília: Organização PanAmericana da Saúde, 2011. 549p. Disponível em: <http://www.abrasco.org.br/UserFiles/File/ABRASCODIVULGA/2011/ RASMendes.pdf> Acesso em: 30 de Junho de 2016.

MIOTO. R. C. T.; NOGUEIRA, V. M. R. Serviço Social e saúde - desafios intelectuais e operativos. Serviço Social, v. 11, n 25, p. 221-243, julho-dez. 2009.

MOREIRA, B.; PELLIZZARO, I. Educação em Saúde: um programa de extensão universitária. Revista Textos \& Contextos. Porto Alegre, v. 8, n. 1, p. 156-171, jan/jun. 2009. Disponível em:

$<$ http://revistaseletronicas.pucrs.br/fo/ojs/index.php/fass/article/view/5680>. Acesso em: 9 de maio de 2014.

MUNARI, D. B. et al. Contribuições para a abordagem da dimensão psicológica dos grupos.

Revista Enfermagem. UERJ, Rio de Janeiro, n. 1, p. 107-112, 2007.

NASCIMENTO, M. L. Baixa escolaridade e sua influência no auto-cuidado da saúde.

Universidade Federal do Triângulo Mineiro. Uberaba, 2014. Monografia (Especialização em Atenção Básica em Saúde da Família). Disponível em:

<http://www.nescon.medicina.ufmg.br/biblioteca/imagem/6348.pdf.> Acesso em: 01 de Dezembro de 2016.

PEREIRA, L. P.; NERY, A. A. Planejamento, gestão e ações à saúde do homem na estratégia de saúde da família. Escola Anna Nery Revista de Enfermagem. Rio de Janeiro, v. 18, n. 4, p. 635-643, Dec. 2014. Disponível em: $<$ http://www.scielo.br/scielo.php?script=sci_arttext\&pid=S141481452014000400635\&lng=e n\&nrm=iso>. Acesso em: 09 de Novembro de 2016. 
ROCHA-COUTINHO, M. L. Novas opções, antigos dilemas: mulher, família, carreira e relacionamento no Brasil. Temas de Psicologia.[online]. 2004, vol.12, n.1, pp. 02-17. ISSN 1413-389X. Disponível em: <http://pepsic.bvsalud.org/pdf/tp/v12n1/v12n1a02.pdf>. Acesso em: 01 de Dezembro de 2016.

SANTOS, L. M. et al. Grupos de promoção à saúde no desenvolvimento da autonomia, condições de vida e saúde. Revista de Saúde Pública. 2006;40(2):346-52. Disponível em: <http://www.scielo.br/pdf/rsp/v40n2/28543.pdf>. Acesso em: 13 de Março de 2016.

SCHRAIBER, L. B. Equidade de Gênero e Saúde: o cotidiano das práticas no Programa de Saúde da Família do Recife. In: VILLELA, W.; MONTEIRO, S. (Org.). Gênero e Saúde: Programa Saúde da Família em questão. Rio de Janeiro, ABRASCO, UNFPA, p. 39-61. 2005.

SOUZA, R. R. A regionalização no contexto atual das políticas de saúde. Ciência Saúde Coletiva (online). 2001;6, n.2:451-55. Disponível em: <www.teses.usp.br/teses/disponiveis/7/7141/tde13012011154823/.../Raquel_Saito.pdf>. Acesso em: 13 de Março de 2016.

VASCONCELOS, E. M. Educação popular: de uma prática alternativa a uma estratégia de gestão participativa das políticas de saúde. Physis (Rio de Janeiro). 2004.

World Health Organization. The Ottawa charter for health promotion. Geneve: WHO; 1986. 
